\title{
Phase Transitions in a Coupled Electron and Spin Model on the Shastry-Sutherland Lattice
}

\author{
P. FARKAŠOVSKÝ, H. ČENČARIKOVÁ* \\ Institute of Experimental Physics SAS, Watsonova 47, 04001 Košice, Slovakia
}

\begin{abstract}
The thermodynamics of a simple electron-spin model proposed recently for a description of magnetization processes in rare-earth tetraborides is studied numerically by the canonical Monte Carlo method in two-dimensions. The model is based on the coexistence of two subsystems, and namely, the spin subsystem described by the Ising model and the electronic subsystem described by the free-electron model on the Shastry-Sutherland lattice (SSL). Moreover, both subsystems are coupled by the anisotropic spin-dependent interaction of the Ising type. At $T=0$ the system exhibits the magnetization plateau (MP) at $m / m_{s}=1 / 2,1 / 3,1 / 5,1 / 7,1 / 9$ and $1 / 11$ of the saturated spin magnetization $m_{s}$. For the largest phases corresponding to $m / m_{s}=0,1 / 3$ and $1 / 2$ we have examined the nature of the phase transitions from the low-temperature ordered phase (LTOP) to the high-temperature disordered phase (HTDP). It is shown that all phases persist also at finite temperatures (up to the critical temperature $T_{c}$ ) and that the phase transition at the critical point is of the second order for the $m / m_{s}=0$ phase and of the first order for the $m / m_{s}=1 / 3$ and $1 / 2$ phases.
\end{abstract}

DOI: $10.12693 /$ APhysPolA.126.44

PACS: 75.10.-b, 75.40.Mg, 05.70.Fh

Recently we have proposed a new model [1] for description of a fascinating sequence of MPs in rare-earth tetraborides observed at fractional values of magnetization, e.g., $m / m_{s}=1 / 2,1 / 9,1 / 11$ in $\mathrm{TmB}_{4}$ [2], $m / m_{s}=1 / 2$, $4 / 9,1 / 3,2 / 9$ and $7 / 9$ in $\mathrm{TbB}_{4}[3], m / m_{s}=1 / 3,4 / 9$ and $3 / 5$ in $\mathrm{HoB}_{4}$ [4]. The model is based on the coexistence of spin and electron subsystems in given materials (with the SSL structure) and the coupling between them. Supposing that these subsystems interact only via the spin dependent Ising interaction $J_{z}$, the Hamiltonian of the system can be written as

$$
\begin{aligned}
H= & \sum_{i j \sigma} t_{i j} d_{i \sigma}^{+} d_{j \sigma}+J_{z} \sum_{i}\left(n_{i \uparrow}-n_{i \downarrow}\right) S_{i}^{z}-h \sum_{i}\left(n_{i \uparrow}-n_{i \downarrow}\right) \\
& +J \sum_{\langle i j\rangle} S_{i}^{z} S_{j}^{z}+J^{\prime} \sum_{\langle\langle i j\rangle\rangle} S_{i}^{z} S_{j}^{z}-h \sum_{i} S_{i}^{z}
\end{aligned}
$$

where $d_{i \sigma}^{+}, d_{i \sigma}$ are the creation and annihilation operators of itinerant electrons in the $d$-band Wannier state at site $i$ and $n_{i \sigma}=d_{i \sigma}^{+} d_{i \sigma}$. The first term of Eq. 1 is the kinetic energy corresponding to the quantum-mechanical hopping of itinerant $d$ electrons between sites $i$ and $j$. These intersite hopping transitions are described by the matrix elements $t_{i j}$, which are $-t$ if $i$ and $j$ are the nearest neighbours, $-t^{\prime}$ if $i$ and $j$ are the next-nearest neighbours and zero otherwise. The second term represents the above mentioned anisotropic, spin-dependent local interaction of the Ising type between the itinerant electrons and localized spins $S_{i}^{z}= \pm 1 / 2$, that also interact mutually via the Ising interaction (the second row of Eq. 1). J, $J^{\prime}$ are the antiferromagnetic exchange coupling between all nearest neighbour bonds $(J)$ and next-nearest neighbour bonds in every second square $\left(J^{\prime}\right)$ of the SSL [5]. The last terms in the first and second row describe action of

*corresponding author; e-mail: hcencar@saske.sk

the magnetic field on the itinerant electrons and localized spins. For this simple model we have found [1], that switching on of the spin-dependent interaction $\left(J_{z}\right)$ between the electron and spin subsystems and taking into account the electron hopping on the nearest $(t)$ and nextnearest $\left(t^{\prime}\right)$ lattice sites of the SSL lead to stabilization of new MPs. In addition, to the Ising MP at $m / m_{s}=1 / 3$ we have found new MPs located at $m / m_{s}=1 / 2,1 / 5$, $1 / 7,1 / 9$ and $1 / 11$ [1], what is in a very good agreement with the experimental measurements in rare-earth tetraborides [2]. In the present paper we extend numerical calculations for this model to non-zero temperatures with a goal to answer the questions about the temperature stability of these phases and the type of phase transitions from the LTOPs to the HTDP. The numerical calculations are done by a straightforward extension of the canonical Monte Carlo method discussed extensively in our previous paper [6]. To identify the transition temperatures from the LTOPs to the HTDP, and the type of the phase transition, we have calculated numerically the specific heat $C=\left(\left\langle E^{2}\right\rangle-\langle E\rangle^{2}\right) /\left(L / \tau^{2}\right)$, the thermal average of the spin occupation $w_{s}=\langle s\rangle$ and the energy distribution $P(E)$, where $\langle E\rangle=\frac{1}{Z} \sum_{s, w^{d}} E e^{-E / \tau}(Z=$ $\left.\sum_{s, w^{d}} e^{-E / \tau}, E=\sum_{i} \varepsilon_{i}(s) w_{i}^{d}\right)$ and summation goes over all distributions of spins $s=\left\{S_{1}^{z}, S_{2}^{z}, \ldots, S_{L}^{z}\right\}$ on a lattice of $L$ sites and over all distributions of electrons $\left(w^{d}\right)$ on single energy levels $\varepsilon_{i}$ of $H\left(\tau=k_{B} T\right)$.

Using this method we have performed exhaustive numerical studies of the model (1) for the following selected model parameters $J_{z}=4, t=4, t^{\prime}=0.4 t, J / J^{\prime}=1$ (for which the best correspondence of theoretical and experimental results has been observed at $\tau=0$ [1]) and selected values of $h$ corresponding to the most interesting physical cases. In particular, we have chosen $h=0.5$ as a representative value for the $m / m_{s}=0$ phase, $h=3$ for the $m / m_{s}=1 / 3$ phase and $h=4.1$ for the $m / m_{s}=1 / 2$ phase. To exclude the finite size effects, the numerical calculations have been done for sev- 
eral SSLs from $L=6 \times 6$ up to $L=18 \times 18$ sites with the periodic boundary conditions. Our numerical results obtained within the canonical Monte Carlo method for $C, w_{s}$ and $P(E)$ are depicted in Fig. 1 .
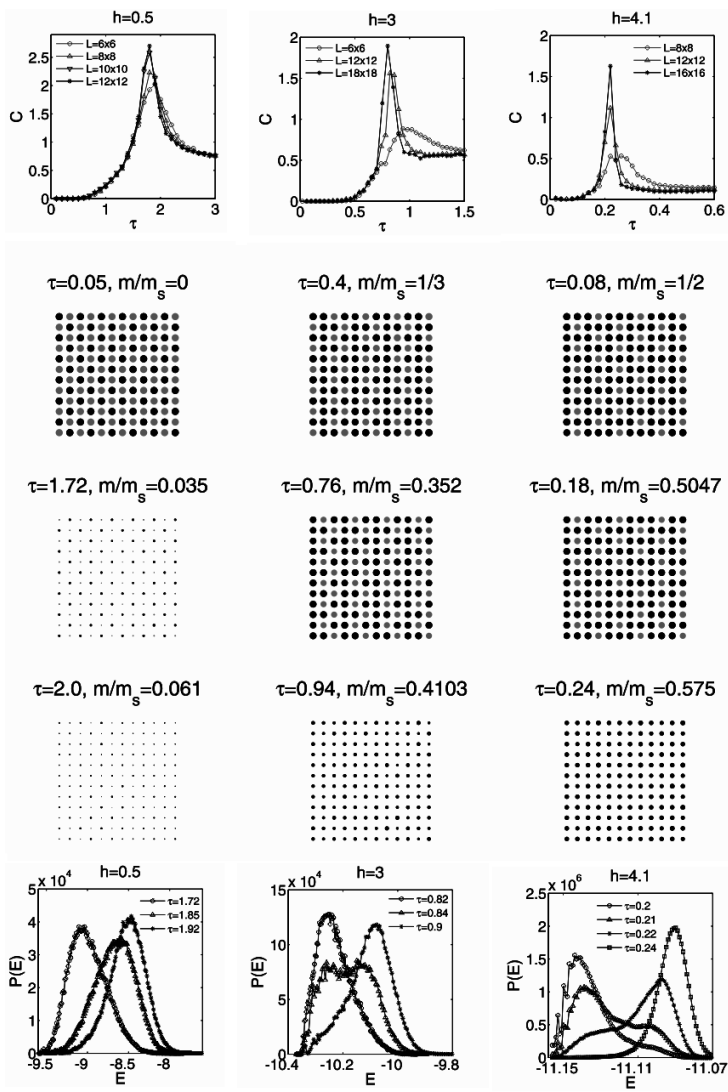

Fig. 1. Row 1: The specific heat as a function of $\tau$ for different values of $h$ and $L$. Rows 2-4: The average spin occupation $w_{s}$ calculated for different values of $\tau, h$ $\left(m / m_{s}\right)$. Black (gray) dots correspond to the up (down) spin orientation. The radius of the circle on site $i$ is proportional to $\left\langle S_{i}^{z}\right\rangle$. Row 5: The energy distribution $P(E)$ as a function of $E$ calculated for different values of $h$ and $\tau$ (near $\tau_{c}$ ).

One can see that, for all selected values of $h$, corresponding to fractional MPs with $m / m_{s}=0,1 / 3$ and $1 / 2$, there exists a sharp low-temperature peak which scales with increasing cluster size $L$, that indicates the presence of the phase transition from the LTOP to the HTDP. In particular, for $h=0.5$ (with an antiferromagnetic ground-state arrangement) we have detected a sharp low-temperature peak located at relatively large value of $\tau \sim 1$.8. This maximum is obviously connected with a transition from the antiferromagnetic chessboard structure to the homogeneous phase. This fact is supported by the behaviour of the average spin occupation $w_{s}$ and the energy distribution $P(E)$ near the transition point $\tau \rightarrow 1.8$. As illustrates the behaviour of $w_{s}$, the antiferromagnetic chessboard structure persists for all temperatures up to the transition point, and the increasing temperature $\tau \rightarrow \tau_{c}$ only resizes the average magnetic moment on each site. At the transition point this type of ordering is fully destroyed. When the temperature is further increasing the homogeneous spin state is detected. The typical examples for representative temperatures, below $\tau_{c}(\tau=0.05$ and 1.72$)$ and above $\tau_{c}(\tau=2)$ are illustrated in Fig. 1. To identify the type of the phase transition, we have calculated the energy distribution $P(E)$ using the method of Challa et al. [7]. One can see that for $h=0.5$ the energy distribution $P(E)$ exhibits the clear one-peak structure and thus the phase transition from the LTOP to the HTDP is the second order phase transition. Thus the fundamental question mentioned above, and namely, if the ground-state arrangements detected for our simple spin-electron model, proposed for a description of rare-earth tetraborides, persist also at non-zero temperatures, was answered positively (at least for the phase $\left.m / m_{s}=0\right)$. The same calculations we have performed also for the case $h=3$ with $m / m_{s}=1 / 3$ and the case $h=4.1$ with $m / m_{s}=1 / 2$. We have found that the transition from the LTOP to the HTDP is of the first order for the case $m / m_{s}=1 / 3$ (with the transition temperature two times smaller in comparison to the $m / m_{s}=0$ case) and of the second order for the $m / m_{s}=1 / 2$ (with the transition temperature approximatively ten times smaller than in the case $m / m_{s}=0$ ).

In summary, we have examined the nature of the phase transitions from the LTOPs to the HTDP in a simple coupled electron-spin model on the SSL. As the lowtemperature ordered phases we have considered the spin structures corresponding to the largest MPs on the magnetization curve (at $m / m_{s}=0,1 / 3$ and $1 / 2$ ). It was shown that all phases persist also at finite temperatures and that the phase transition at the critical point is of the second order for the $m / m_{s}=0$ phase and of the first order for the $m / m_{s}=1 / 3$ and $1 / 2$ phases.

\section{Acknowledgments}

This work was supported by the VEGA grant No.2/0077/13, the APVV grant No. APVV-0097-12 and the ERDF EU grant No. ITMS.26220120005.

\section{References}

[1] P. Farkašovský, H. Čenčariková, S. Mataš, Phys. Rev. B 82, 054409 (2010).

[2] K. Siemensmeyer, E. Wulf, H.-J. Mikeska, K. Flachbart, S. Gabáni, S. Mat'aš, P. Priputen, A. Efdokimova, N. Shitsevalova, Phys. Rev. Lett. 101, 177201 (2008).

[3] S. Yoshii, T. Yamamoto, M. Hagiwara, S. Michimura, A. Shigekawa, F. Iga, T. Takabatake and K. Kindo, Phys. Rev. Lett. 101, 087202 (2008).

[4] S. Matas, K. Siemensmeyer, E. Wheeler, E. Wulf, R. Beyer, Th. Hermannsdörfer, O. Ignatchik, M. Uhlarz, K. Flachbart, S. Gabáni, P. Priputen, A. Efdokimova and N. Shitsevalova, J. Phys.: Conf. Ser. 200, 032041 (2010).

[5] B.S. Shastry and B. Sutherland, Physica $B$ and $C$ 108, 1069 (1981).

[6] H. Čenčariková, P. Farkašovský, Eur. Phys. J. B 7, 393 (2010).

[7] M.S.S. Challa, D.P. Landau, K. Binder Phys. Rev. B 34, 1841 (1986). 